\title{
How ATP-Competitive Inhibitors Allosterically Modulate Tyrosine Ki- nases That Contain a Src-like Regulatory Architecture
}

\author{
Linglan Fang ${ }^{\dagger}$, Jessica Vilas-Boas ${ }^{\ddagger}$, Sujata Chakraborty ${ }^{\dagger}$, Zachary E. Potter ${ }^{\dagger}$, Ames C. Register ${ }^{\dagger}$, Markus \\ A. Seeliger ${ }^{\ddagger}$, and Dustin J. Maly ${ }^{\dagger}, *$ \\ tDepartment of Chemistry and ${ }^{\S}$ Biochemistry, University of Washington, Seattle, Washington 98125, USA \\ tDepartment of Pharmacological Sciences, Stony Brook University, Stony Brook, NY 11794-8651, USA
}

\begin{abstract}
Small molecule kinase inhibitors that stabilize distinct ATP-binding site conformations can differentially modulate the global conformation of Src-family kinases (SFKs). However, it is unclear which specific ATP-binding site contacts are responsible for modulating the global conformation of SFKs and whether these inhibitor-mediated allosteric effects are general to other tyrosine kinases. Here, we describe the development of chemical probes that allow us to deconvolute which features in the ATP-binding site are responsible for the allosteric modulation of the global conformation of Src. We find that the ability of an inhibitor to modulate the global conformation of Src's regulatory domain-catalytic domain module relies mainly on the influence it has on the conformation of a structural element called helix $\alpha$ C. Furthermore, by developing a set of orthogonal probes that target a drug-sensitized Src variant, we show that stabilizing Src's helix $\alpha \mathrm{C}$ in an active conformation is sufficient to promote a Src-mediated, phosphotransferase-independent alteration in cell morphology. Finally, we report that ATP-competitive, conformation-selective inhibitors can influence the global conformation of tyrosine kinases beyond the SFKs, suggesting that the allosteric networks we observe in Src are conserved in kinases that have a similar regulatory architecture. Taken together, our study highlights that an ATP-competitive inhibitor's interactions with helix $\alpha \mathrm{C}$ can have a major influence on the global conformation of some tyrosine kinases in vitro and in cells.
\end{abstract}

The human genome encodes $\sim 540$ eukaryotic protein kinases, an indication of the vast number of kinase-mediated cellular functions. ${ }^{1}$ All kinases contain at least one highly homologous catalytic domain (CD) that is of similar overall architecture. Almost half of all protein kinases contain at least one auxiliary domain outside of the $\mathrm{CD}^{2}$ Precise regulation of these multidomain kinases often relies on intramolecular interactions between the $\mathrm{CD}$ and auxiliary domains. Intramolecular regulation of kinase phosphotransferase activity is pervasive - regulatory domains can allosterically modulate phosphotransferase activity by engaging and suppressing the CD intramolecularly. ${ }^{3-4}$ In many cases, the global conformational changes within these regulatory domains also facilitate important phosphotransferase-independent functions in cells, including regulating protein scaffolding, localization, and gene transcription. ${ }^{5-7}$

Src-family kinases (SFKs) are one of the best-characterized multidomain kinase families. ${ }^{8-11}$ SFKs contain a membrane-interacting SH4 domain, a unique domain, and regulatory $\mathrm{SH} 2$ and $\mathrm{SH} 3$ domains that modulate the phosphotransferase activities of their CDs. Domains outside of the CD also provide additional binding surfaces for facilitating protein-protein interactions (Figure 1A). ${ }^{12-13}$ In the inactive form, SFKs adopt a closed global conformation, where the phosphotransferase activity of the CD is allosterically suppressed by multiple intramolecular interactions: the SH3 domain's interaction with the SH2-CD linker, the SH2 domain's interaction with the phosphorylated C-terminal tail, and the SH4 domain's interaction with the Clobe of the CD. Upon activation, these autoinhibitory, intramolecular interactions are released and SFKs adopt a more open global conformation with enhanced phosphotransferase activity. $^{14-15}$

Interdomain regulatory interactions suppress the phosphotransferase activity of SFKs by allosteric stabilization of an inactive conformation of the ATP-binding site, which misaligns the key catalytic residues for catalysis. ${ }^{7,}{ }^{16} \mathrm{We}$ and others have shown that ATP-competitive inhibitors that stabilize different ATP-binding site conformations can exploit these allosteric networks to modulate the regulatory interactions of the $\mathrm{SH} 3-\mathrm{SH} 2-$ CD module of SFKs. ${ }^{14,}{ }^{17-23}$ Inhibitors that stabilize the helix $\alpha \mathrm{C}$-out inactive conformation of the ATP-binding site, which involves outward movement of the catalytically important helix $\alpha \mathrm{C}$ (Figure 1B), promote an autoinhibited, closed global conformation of the SH3-SH2-CD module of SFKs. The presence of an extended substituent that occupies the hydrophobic pocket formed by the displacement of helix $\alpha \mathrm{C}$ is necessary for inhibitors to stabilize this inactive conformation. In contrast, inhibitors that stabilize the DFG-out inactive ATP-binding site conformation, which involves the displacement of the DFG-motif in the activation loop, promote an open, regulatory domain disengaged conformation of the SH3-SH2-CD module (Figure 1C). Despite extensive biochemical and structural characterization, the ATP-binding site contacts that are directly responsible for the divergent effects that helix $\alpha \mathrm{C}$-out- and DFG-outstabilizing inhibitors have on the global conformations of SFKs are not completely known.

It is also unclear how general the effects of conformation-selective inhibitors are on kinase conformation beyond the SFKs. 

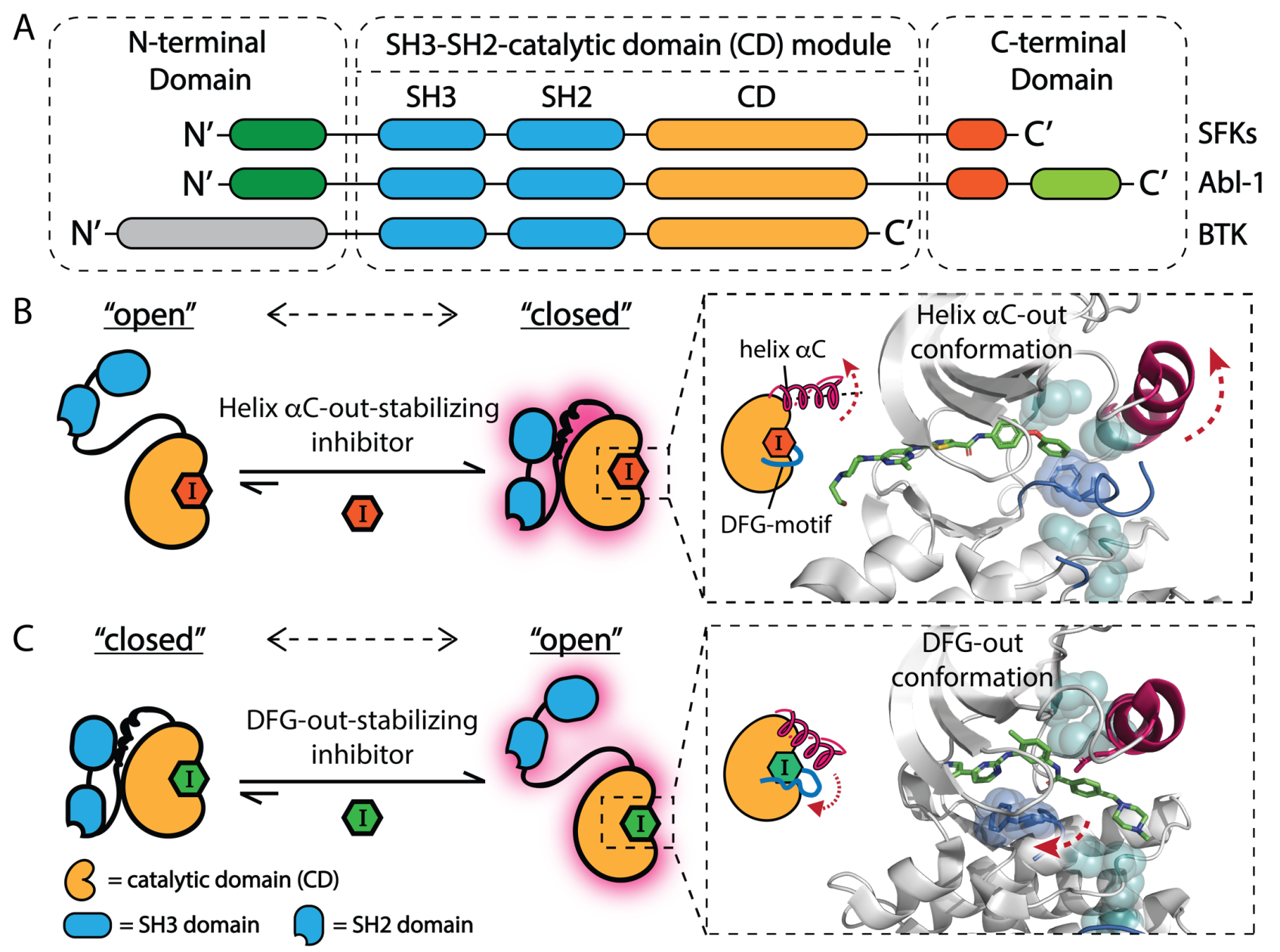

Figure 1. Allosteric modulation of tyrosine kinases that contain an SH3-SH2-catalytic domain (CD) module. (A) Domain arrangement of Src-family kinases (SFKs), Abl-1, and BTK. (B-C) ATP-competitive, conformation-selective inhibitors can modulate the global conformation of SFKs. (B) Helix $\alpha$ C-out-stabilizing inhibitors promote a closed global conformation of Src's SH3-SH2-CD module by stabilizing the ATP-binding site in the helix $\alpha \mathrm{C}$-out form (PDB: 4YBK). ${ }^{23}$ Shown are the catalytic spine (cyan), helix $\alpha \mathrm{C}$ (magenta), and the activation loop (blue). (C) DFG-out-stabilizing inhibitors promote an open global conformation of Src's SH3-SH2-CD module by stabilizing the ATPbinding site in the DFG-out conformation (PDB: 2OIQ). ${ }^{24}$

Structural analysis of the Abelson tyrosine kinase 1 (Abl-1), which contains a Src-like SH3-SH2-CD module, have demonstrated that ATP-competitive inhibitors can also modulate the global conformation of Abl-1. ${ }^{25-28}$ Specifically, the ability of inhibitors to flip the DFG-motif in the activation loop correlates with their abilities to promote an open global conformation of Abl-1's SH3-SH2-CD module. ${ }^{29}$ Beyond the SFKs and Abl$1 / \mathrm{Arg}$, there are at least 10 additional tyrosine kinases that contain an SH3-SH2-CD module (Figure S1) ${ }^{30}$ It is currently unknown whether inhibitors that stabilize the DFG-out and helix $\alpha \mathrm{C}$-out inactive ATP-binding site conformations can also allosterically modulate the global conformation of SH3-SH2-CD modules of tyrosine kinases beyond the SFKs.

In this study, we describe the development of chemical probes that allow us to deconvolute which features in the ATPbinding site are responsible for the allosteric modulation of the SH3-SH2-CD module of Src. With these probes, we found that the ability of an inhibitor to allosterically modulate the global conformation of Src's SH3-SH2-CD module depends mainly on its influence on the conformation of helix $\alpha \mathrm{C}$. Thus, the observed ability of DFG-out-stabilizing inhibitors to promote an open global conformation of Src is not due to their influence on the DFG-motif in the activation loop but rather their ability to stabilize an active conformation of helix $\alpha \mathrm{C}$. Using these design principles, we developed a new probe that can be used with a drug-sensitized Src variant to specifically stabilize Src's helix $\alpha \mathrm{C}$ in the active conformation without perturbing its activation loop. Using this chemical genetic approach, we found that stabilizing Src's helix $\alpha \mathrm{C}$ in an active conformation was sufficient to promote a Src-dependent phosphotransferase-independent alteration in cell morphology. Finally, we report that ATPcompetitive, conformation-selective inhibitors can divergently modulate the global conformation of the SH3-SH2-CD module of tyrosine kinases beyond the SFKs. Taken together, our biochemical and cellular data strongly suggest that the ability of inhibitors to influence the conformation of helix $\alpha \mathrm{C}$ is the main molecular determinant for allosterically modulating the global conformation of tyrosine kinases that contain an SH3-SH2-CD module.

\section{RESULTS AND DISCUSSION}

Inhibitors divergently modulate the global conformation of tyrosine kinases that contain an SH3-SH2-CD module. Prior to exploring the molecular details of how inhibitors influence the global conformation of the SH3-SH2-CD module, we first explored whether the divergent allosteric modulation we observed with the SFKs extended beyond this kinase family. To 
explore the pervasiveness of this effect, we characterized how stabilizing the DFG-out and helix $\alpha \mathrm{C}$-out ATP-binding site conformations affect the regulatory domains of Abl-1, the best-

A helix $\alpha \mathrm{C}$ Helix $\alpha \mathrm{C}$-out pobe
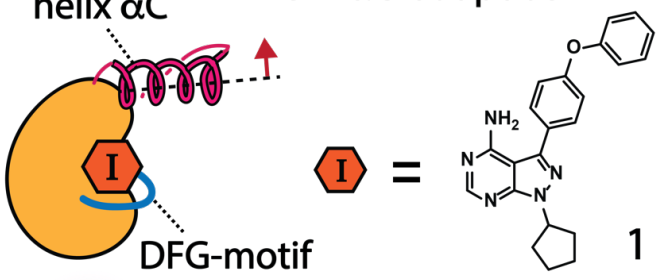

characterized member of Abl family kinases, and BTK, a Tec family kinase.
B

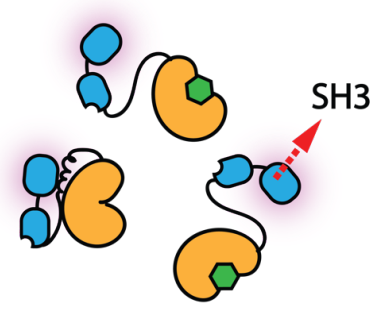

C

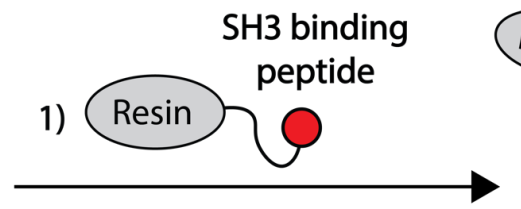

2) Extensive washing
DFG-out pobe

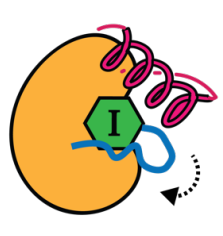<smiles>Cc1cc[nH]c1C=Cc1nnc(O)n1C=Cc1cccc(C(=O)NC=C=O)c1</smiles>

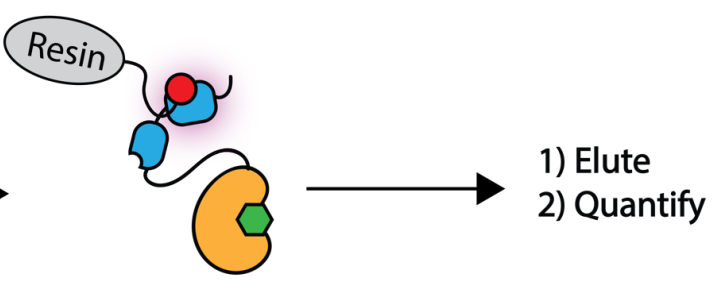

Intermolecular accessibility

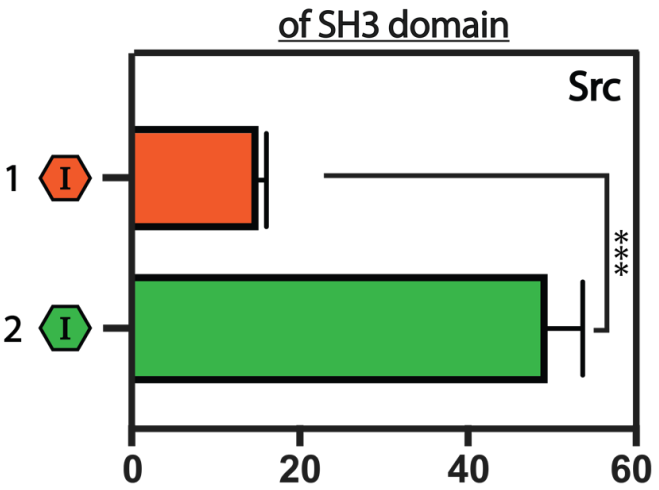

D
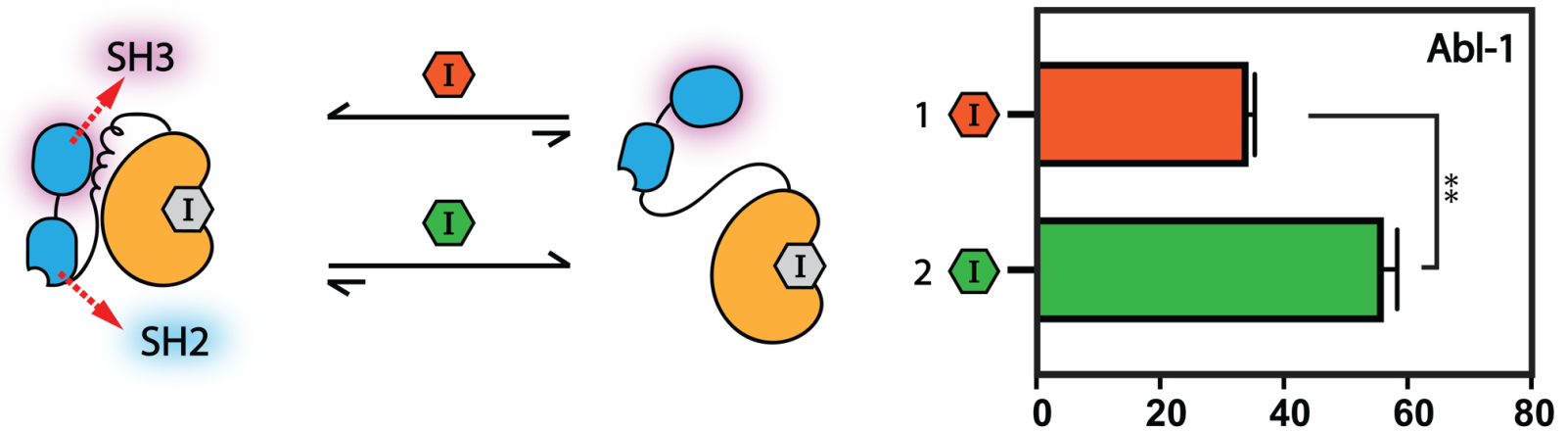

E
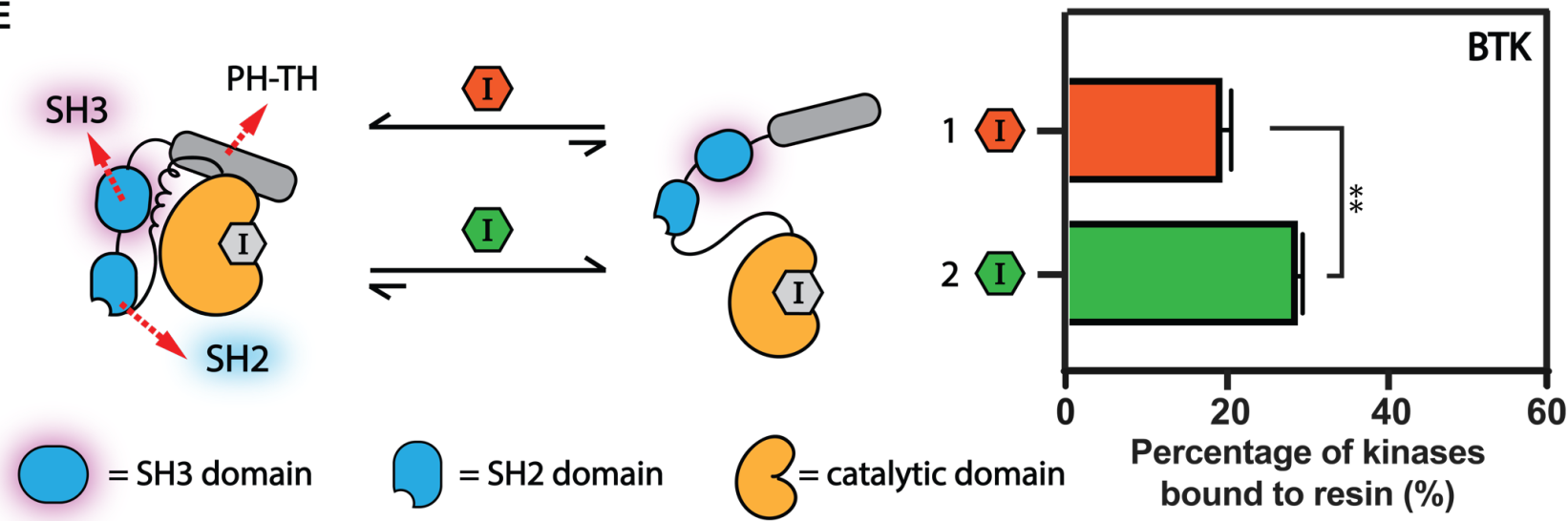

Figure 2. Conformation-selective inhibitors divergently modulate the global conformation of multidomain tyrosine kinases that contain an SH3-SH2-CD module. (A) Helix $\alpha$ C-out-stabilizing inhibitor 1 (left) and DFG-out-stabilizing inhibitor 2 (right). (B) An illustration of the SH3 domain pull-down assay. (C-E) Determination of the global conformation of inhibitor-bound tyrosine kinases that contain the SH3- 
SH2-CD modules. (C) Allosteric modulation of Src's SH3-SH2-CD module by conformation-selective inhibitors. Inhibitor 1 promotes a closed SH3-SH2-CD module of Src. Inhibitor 2 promotes an open SH3-SH2-CD module of Src. (D) Allosteric modulation of Abl-1's SH3SH2-CD module by conformation-selective inhibitors. Inhibitor 2 promotes a more open conformation of Abl-1's SH3-SH2-CD module than inhibitor 1. (E) Allosteric modulation of BTK's SH3-SH2-CD module by conformation-selective inhibitors. Inhibitor 1 promotes a more closed conformation of BTK's SH3-SH2-CD module than inhibitor 2. All values shown are mean \pm SEM ( $\mathrm{n}=3$ ). P-values are calculated using a two-tailed t-test $(*<0.05, * *<0.01, * * *<0.001)$.

To modulate the ATP-binding sites of tyrosine kinases, we generated two inhibitors that stabilize the helix $\alpha \mathrm{C}$-out and DFG-out inactive conformations, respectively. Inhibitor 1 contains an extended 4-phenoxyphenyl substituent at the C-3 position on the pyrazolopyrimidine scaffold that occupies a hydrophobic pocket created by the outward movement of helix $\alpha \mathrm{C}$ (Figure 2A, left $).{ }^{14,18-19,31}$ Inhibitor 2 stabilizes the DFG-out inactive conformation of tyrosine kinases (Figure $\mathbf{2 A}$, right). Inhibitor $\mathbf{2}$ contains a 3-trifluoromethylbenzamide substituent that occupies the hydrophobic pocket formed by the displacement of the DFG-motif. ${ }^{14}$, 32 The conformationally rigid alkynylphenyl linker of $\mathbf{2}$ is most likely only compatible with DFG-out inactive conformation. Since $\mathbf{1}$ and $\mathbf{2}$ only differ at their C-3 substituents, the effects on the regulatory domains of tyrosine kinases should be due to each inhibitor stabilizing the desired inactive ATP-binding site conformation.

We first validated that each inhibitor biochemically modulates Src's SH3-SH2-CD module as expected by performing SH3 domain pull-down assays with an immobilized SH3 domain ligand and inhibitor-bound Src complexes (Figure 2B). As expected, we found that the $\mathrm{SH} 3$ domain of the $\mathrm{Src} / \mathbf{1}$ complex was inaccessible to intermolecular engagement with the SH3 domain ligand, consistent with helix $\alpha \mathrm{C}$-out-stabilizing inhibitors promoting a closed, autoinhibited SH3-SH2-CD module (Figure 2C). Conversely, the $\mathrm{Src} / \mathbf{2}$ complex was significantly enriched by the immobilized SH3 domain ligand, which is consistent with $\mathbf{2}$ promoting a regulatory domain disengaged conformation of Src's SH3-SH2-CD module. Thus, inhibitors 1 and 2 can divergently modulate the Src SH3-SH2-CD module by stabilizing two distinct inactive ATP-binding site conformations.

We then investigated how our conformation-selective inhibitors affect Ab1-1's SH3-SH2-CD module. We first compared the SH3 domain accessibility of the Abl-1/1 and Abl-1/2 complexes. Like Src, Abl-1/2 complex adopts a more open global conformation within the SH3-SH2-CD module, with a more intermolecularly accessible SH3 domain, relative to the Abl-1/1 complex (Figure 2D). To further validate how stabilization of Abl-1 in both the helix $\alpha$ C-out and DFG-out conformations affects the global conformation of its $\mathrm{SH} 3-\mathrm{SH} 2-\mathrm{CD}$ module, we utilized electrophile-containing analogs of $\mathbf{1}$ and $\mathbf{2 - 1} \mathbf{a}$ and $\mathbf{2 a}$, respectively - that potently inhibit a drug-sensitized cysteine mutant of Abl-1 (Abl-1 V256C) (Figure S2A). As expected, we found that the SH3 domain of the Abl-1 V256C/1a complex was largely inaccessible to intermolecular SH3 ligand engagement, which is consistent with helix $\alpha \mathrm{C}$-out-stabilizing 1a promoting a closed conformation of Abl-1 V256C's SH3-SH2-CD module (Figure S2B,S2C). Conversely, the SH3 domain of Abl-1 V256C/2a complex was efficiently enriched by the immobilized SH3 ligand, consistent with DFG-out stabilizing inhibitor 2a promoting an open, intramolecularly disengaged Abl1 SH3-SH2-CD module, like its underivatized counterpart. Thus, consistent with previous observations, ${ }^{25,}{ }^{29}$ an inhibitor that stabilizes the DFG-out inactive ATP-binding site conformation significantly promotes a regulatory domain-disengaged conformation of Abl-1's SH3-SH2-CD module. Inhibitors that

stabilize the helix $\alpha \mathrm{C}$-out inactive ATP-binding site conformation promote a closed Abl-1's SH3-SH2-CD module, which further highlights the Src-like allosteric communication between Abl-1's regulatory domains and its ATP-binding site.

Next, we explored whether conformation-selective, ATPcompetitive inhibitors can also divergently modulate BTK's SH3-SH2-CD module. We first evaluated how the SH3 domain accessibility of the BTK $/ \mathbf{1}$ complex compared to the BTK $/ 2$ complex. Like Src and Abl-1, helix $\alpha \mathrm{C}$-out-stabilizing inhibitor 1 resulted in a closed global conformation of BTK's SH3-SH2CD module, with a largely intermolecularly inaccessible SH3 domain relative to the BTK/2 complex (Figure 2E). To further validate how stabilization of BTK in both inactive ATP-binding site conformations affects the global conformation of its SH3SH2-CD module, we used a drug-sensitized variant of BTK (BTK V416C) with inhibitors 1a and 2a. ${ }^{13-14}$ Like wild-type BTK, we found that the SH3-SH2-CD module of the BTK V416C/1a complex adopted a more closed global conformation compared to the BTK/2a complex (Figure S3). Thus, conformation-selective inhibitors are capable of divergently modulating BTK's SH3-SH2-CD module, based on the ATP-binding site conformation they stabilize.

Binding features of inhibitors that promote an open global conformation of Src. While inhibitors that stabilizes the DFG-out inactive conformation promote an open global conformation of tyrosine kinases that contain an SH3-SH2-CD architecture, ${ }^{22,23}$ we have found that other classes of ATPcompetitive inhibitors are also capable of promoting allosteric disengagement of regulatory $\mathrm{SH} 2$ and $\mathrm{SH} 3$ domains. ${ }^{18}$ To better understand the common features of inhibitors that promote an open global conformation of the $\mathrm{SH} 3-\mathrm{SH} 2-\mathrm{CD}$ regulatory module, we assembled a panel of 13 inhibitors that contain structurally diversified substituents that make varied ATP-binding site contacts and tested them in biochemical assays for the global conformational state of Src (Figure 3A). Inhibitors 1, 3, and 4 contain pharmacophores that should stabilize the helix $\alpha \mathrm{C}$-out inactive conformation of Src, ${ }^{14,17}$ while $\mathbf{2}$ and $\mathbf{5}$ contain substituents that stabilize the DFG-out form. ${ }^{14,32}$ The other eight inhibitors in our panel (6-13) contain various substituents that project into the ATP-binding pocket but are predicted to not stabilize either inactive conformation of the ATP-binding site.

To probe how our panel of inhibitors modulate the global conformation of Src's SH3-SH2-CD module, we performed limited proteolysis experiments with inhibitor-bound Src complexes (Figure 3B,3C). ${ }^{22}$ It has previously been demonstrated that the metalloprotease thermolysin can selectively cleave the flexible linker that connects Src's SH2 domain to its CD (SH2$\mathrm{CD}$ linker) and that the cleavage rate is sensitive to Src's global regulatory conformation (Figure 3B, colored in magenta). ${ }^{22,} 33$ Therefore, we used half-life values for proteolytic cleavage as readouts for the global conformation of Src's SH3-SH2-CD module. We benchmarked the half-life for cleavage of apo Src as $33 \pm 3 \mathrm{~min}$. To assign a global conformation to inhibitorbound Src complexes, we measured the half-life for their cleavage and used $5 \sigma$ as the cutoff for significant deviation from apo Src (Figure 3D). Consistent with the ability of DFG-out- 
stabilizing inhibitors to disengage the regulatory $\mathrm{SH} 2$ and $\mathrm{SH} 3$ domains from Src's CD, the SH2-CD linkers of the Src/2 and $\mathrm{Src} / \mathbf{5}$ complexes are cleaved more rapidly than the apo Src. Conversely, consistent with helix $\alpha \mathrm{C}$-out-stabilizing inhibitors enhancing the regulatory $\mathrm{SH} 2$ and $\mathrm{SH} 3$ domain engagement with Src's CD, the SH2-CD linkers of the $\mathrm{Src} / \mathbf{1}, \mathrm{Src} / \mathbf{3}$, and Src/4 complexes are cleaved more slowly than the apo Src.

A

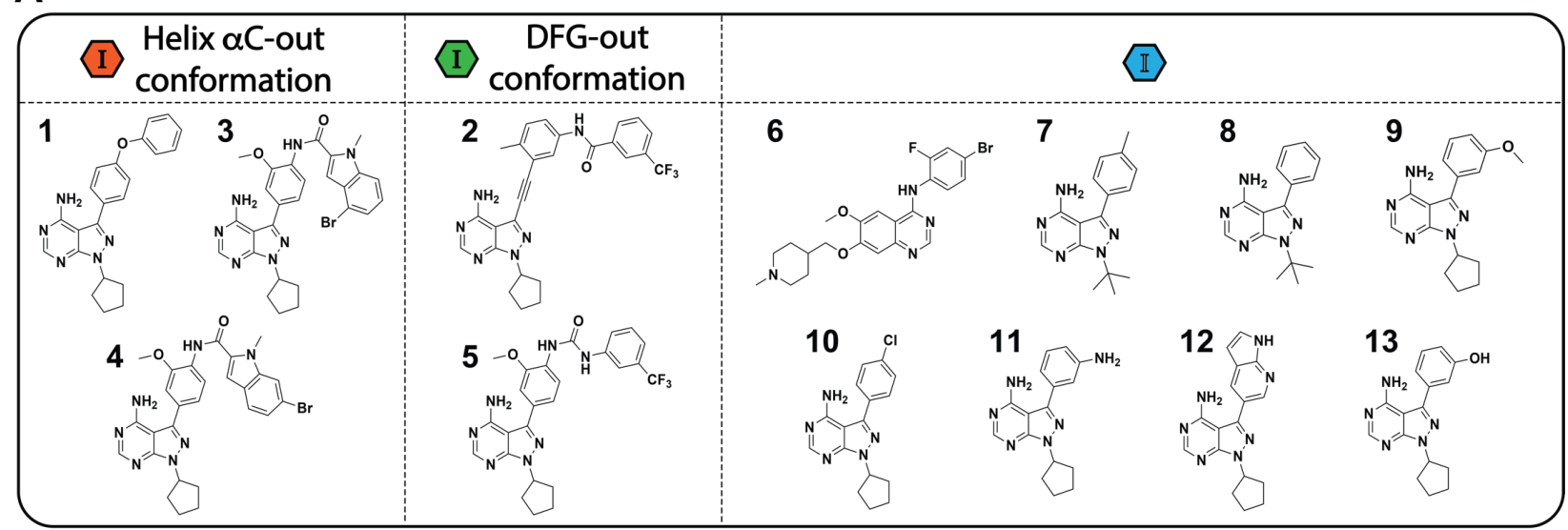

B

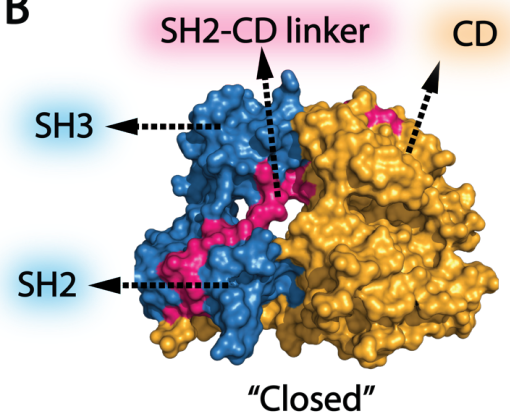

D

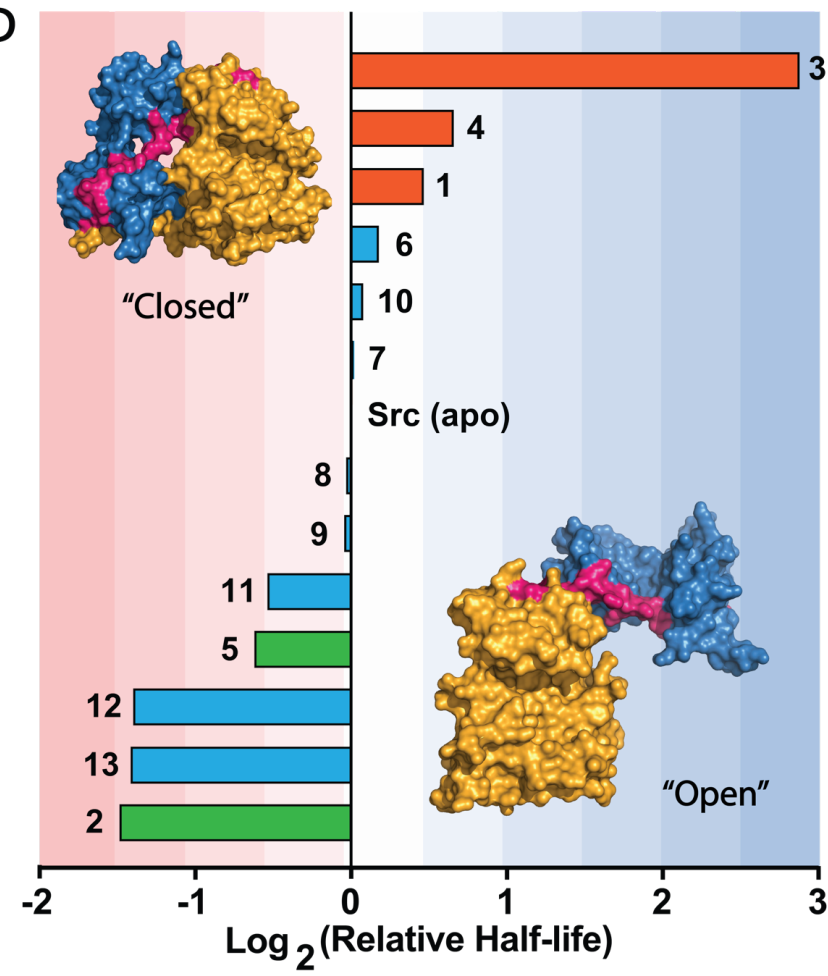

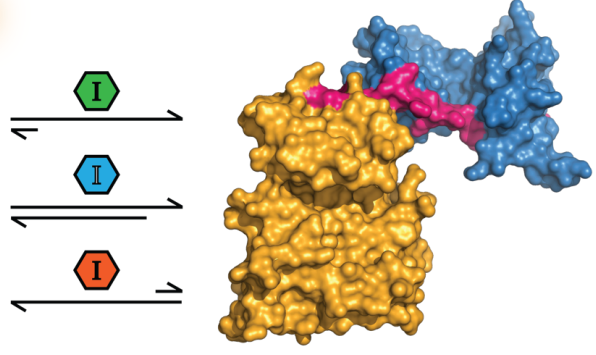

"Open"

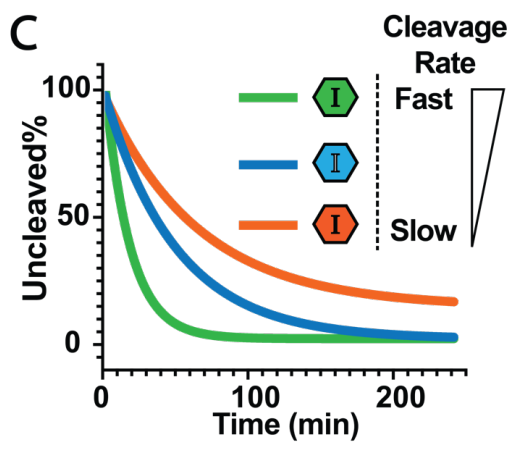

Half Life (min)

Compound Inhibitor Type

\begin{tabular}{ccc}
\hline $250 \pm 40$ & 3 & I \\
\hline $53 \pm 4$ & 4 & I \\
\hline $46 \pm 4$ & 1 & I \\
\hline $38 \pm 2$ & 6 & I \\
\hline $35 \pm 7$ & 10 & I \\
\hline $34 \pm 4$ & 7 & I \\
\hline $33 \pm 3$ & DMSO & Apo \\
\hline $33 \pm 1$ & 8 & I \\
\hline $32 \pm 2$ & 9 & I \\
\hline $23 \pm 3$ & 11 & I \\
\hline $22 \pm 3$ & 5 & I \\
\hline $13 \pm 1$ & 12 & I \\
\hline $12 \pm 1$ & 13 & I \\
\hline $12 \pm 1$ & 2 & I \\
\hline
\end{tabular}

Figure 3. Binding features of inhibitors that promote an open global conformation of Src. (A) A panel of 13 ATP-competitive Src inhibitors. (B) Allosteric modulation of Src's global conformation with ATP-competitive inhibitors. (C) Thermolysin assay. The rate for proteolytic cleavage of the SH2-CD linker is sensitive to the global conformation of inhibitor-bound Src complexes. Thermolysin cleaves the SH2-CD linker (magenta) of inhibitor-bound $\mathrm{Src}$ in the open global conformation more quickly relative to the closed global conformation. The catalytic domain (CD) is colored in yellow and regulatory domains in blue. (D) (Left) Ranking of the log2-based relative half-life values of 
proteolytic cleavage rates of Src's SH2-CD linker. Relative ratio= $\log _{2}$ [Half-life(Inhibitor-bound Src)/Half-life(apo Src)]. The means of the relative ratios are shown. (Right) The mean \pm SEM of half-life values are shown $(n=3)$.

As expected, we found that the SH2-CD linker of Src bound to inhibitors $\mathbf{6}, \mathbf{7}, \mathbf{8}, \mathbf{9}$, or $\mathbf{1 0}$, which all contain substituents that should make minimal interactions with helix $\alpha \mathrm{C}$ or the activation loop, was cleaved at rates similar to Src's apo form (Figure 3D). However, although 11, 12, and 13 do not contain substituents that stabilize either inactive conformation of Src's ATPbinding site, the SH2-CD linker of Src bound to these inhibitors was cleaved at rates similar to the $\mathrm{Src} / \mathbf{2}$ and $\mathrm{Src} / \mathbf{5}$ complexes. Thus, 11-13 appear to promote a regulatory domain disengaged conformation of the SH3-SH2-CD module like DFG-outstabilizing inhibitors. Despite lacking a substituent that promotes a flipped DFG-motif, like DFG-out stabilizing inhibitors 2 and 5, 11-13 contain H-bond donors that we predicted are capable of forming a $\mathrm{H}$-bonding interaction with the side-chain of Glu310 in Src's helix $\alpha$ C. As most inhibitors that stabilize the DFG-out conformation also contain $\mathrm{H}$-bond donors that form a H-bonding interaction with the side-chain of Glu310 in Src's helix $\alpha \mathrm{C}$ (Figure 4A), we further investigated whether this interaction is responsible for promoting an open global conformation of the SH3-SH2-CD module rather than their influence on the activation loop.

Analysis of how helix $\alpha \mathbf{C}$ interactions influence global conformation. Recent studies have shown that the ability of inhibitors to flip the DFG-motif of Abl-1's activation loop highly correlates with their promotion of an open global conformation of Abl-1's SH3-SH2-CD module. ${ }^{29}$ Our observations in Src made us interested in revisiting the molecular binding features of 14 ligands that have previously been reported to promote an open global conformation of Abl-1, which was determined by characteristic NMR ${ }^{1} \mathrm{H}_{-}{ }^{15} \mathrm{~N}$ backbone chemical shifts within the SH3 and SH2 domains (Figure S4). ${ }^{29}$ We found that all inhibitors - including imatinib, nilotinib, ponatinib, rebastinib, and bafetinib - that promote an open global conformation of Abl1's SH3-SH2-CD module also form a H-bonding interaction with the side-chain of Abl-1's Glu286 that stabilize its helix $\alpha \mathrm{C}$ in the active conformation (Figure S5). In contrast, inhibitors that lack the ability to form a H-bonding interaction with Glu286 do not promote an open global conformation of Abl-1 (Figure S4B). Taken together, an inhibitor's ability to hydrogen bond with the conserved Glu in helix $\alpha \mathrm{C}$ highly correlates with the global conformation of Src's and Abl-1's SH3-SH2-CD modules. Therefore, we speculate that the influence of an inhibitor on the conformation of helix $\alpha \mathrm{C}$, and not on the DFG-motif, is responsible for its ability to promote an open global conformation of the SH3-SH2-CD module.

To test this notion, we designed inhibitors that possess the same H-bonding pattern as DFG-out-stabilizing inhibitors but lack bulky substituents that flip the DFG motif of the activation loop. We generated two analogs-14 and $\mathbf{1 5}$ (Figure 4B) - of DFG-out stabilizing inhibitor $\mathbf{2}$, which contains an amide linker that is predicted to hydrogen bond with Glu310 on helix $\alpha \mathrm{C}$ and a bulky trifluoromethylphenyl group that is capable of occupying the hydrophobic pocket created by the displacement of the phenylalanine side-chain in the DFG-motif (Figure S6). ${ }^{14,32} 14$ and 15 retain the same $\mathrm{H}$-bonding pattern as $\mathbf{2}$ but contain less bulky alkyl substituents. While, unsurprisingly, $\mathbf{1 4}$ and $\mathbf{1 5}$ are less potent inhibitors than $\mathbf{2}$, they both inhibit the phosphotransferase activity of Src and Abl-1 at reasonable concentrations.

To determine whether our new analogs interact with the ATP-binding site of Src as designed, we obtained a crystal structure of 14 bound to the CD of Src (Figure 4C and Figure S7). Two molecules of 14-bound Src's CD were observed per crystallographic asymmetric unit. Inhibitor 14 occupies the ATP-binding site of Src and makes the same H-bonding interactions with the hinge region as similar pyrrolopyrimidinebased inhibitors. ${ }^{14,32,34-36}$ Notably, the Src/14 complex is in the active conformation, which is characterized by an inward position of the helix $\alpha \mathrm{C}$ (helix $\alpha \mathrm{C}$-in) in the $\mathrm{N}$-lobe and an unperturbed activation loop. Like a DFG-out stabilizing inhibitor, the amide linker that projects from the alkynylphenyl group of $\mathbf{1 4}$ forms a H-bond with Glu310 in the helix $\alpha \mathrm{C}$, presumably stabilizing the active conformation of this structural element (Figure S7). Although the acetamide group of 14 projects toward the activation loop, Src's DFG-motif is not flipped. An overlay of the Src/14 complex with a crystal structure of Src in the DFG-out conformation shows that $\mathbf{1 4}$ 's acetamide group is not large enough to perturb the Phe 405 residue of the DFG-motif in the activation loop from an active conformation.

To confirm that inhibitors $\mathbf{1 4}$ and $\mathbf{1 5}$ have a similar effect on Src's ATP-binding site in solution, we monitored their binding kinetics with Src using a previously reported stopped-flow tryptophan fluorescence assay (Figure 4D). For inhibitors that stabilize the DFG-out conformation, the rate-determining event for binding at high inhibitor concentrations is the flipping of the DFG-motif. This leads to an observed rate constant for binding $\left(k_{o b s}\right)$ that deviates from linearity of otherwise pseudo first-order kinetics. ${ }^{37-38}$ Consistent with the flipping of the DFG-motif not being required for $\mathbf{1 4}$ and $\mathbf{1 5}$ to interact with the ATP-binding site of Src, we found that the observed rate constants for both inhibitors increased linearly with concentration (Figure 4D, colored in yellow; Figure S8A,S8B). In contrast, inhibitor 16, which is a direct DFG-out-stabilizing analog of $\mathbf{1 4}$ and $\mathbf{1 5}$, demonstrated a nonlinear $k_{o b s}$ with increasing inhibitor concentration, consistent with $\mathbf{1 6}$ requiring a flipped DFG-motif to be accommodated in Src's ATP-binding site (Figure 4D, colored in green). Additionally, because the flip of the DFG-motif necessitates protonation of the side-chain of its Asp residue, the kinetics of DFG-out-stabilizing inhibitors are $\mathrm{pH}$-dependent. ${ }^{37}$ ${ }^{38}$ We found that the observed rate constants for binding of $\mathbf{1 4}$ and $\mathbf{1 5}$ to Src were not dependent on $\mathrm{pH}$, while the binding of $\mathbf{1 6}$ to Src was (Figure S8C-F). We also tested $\mathbf{1 4}$ and $\mathbf{1 5}$ for their binding kinetics with Abl-1 and found that both inhibitors demonstrate a kinetic profile that is similar to their interaction with Src (Figure S9). Taken together, we validated that inhibitor $\mathbf{1 4}$ and $\mathbf{1 5}$ retain the same $\mathrm{H}$-bonding pattern as DFG-outstabilizing inhibitors but do not require flipping of the DFGmotif in the activation loop of tyrosine kinases for binding. 


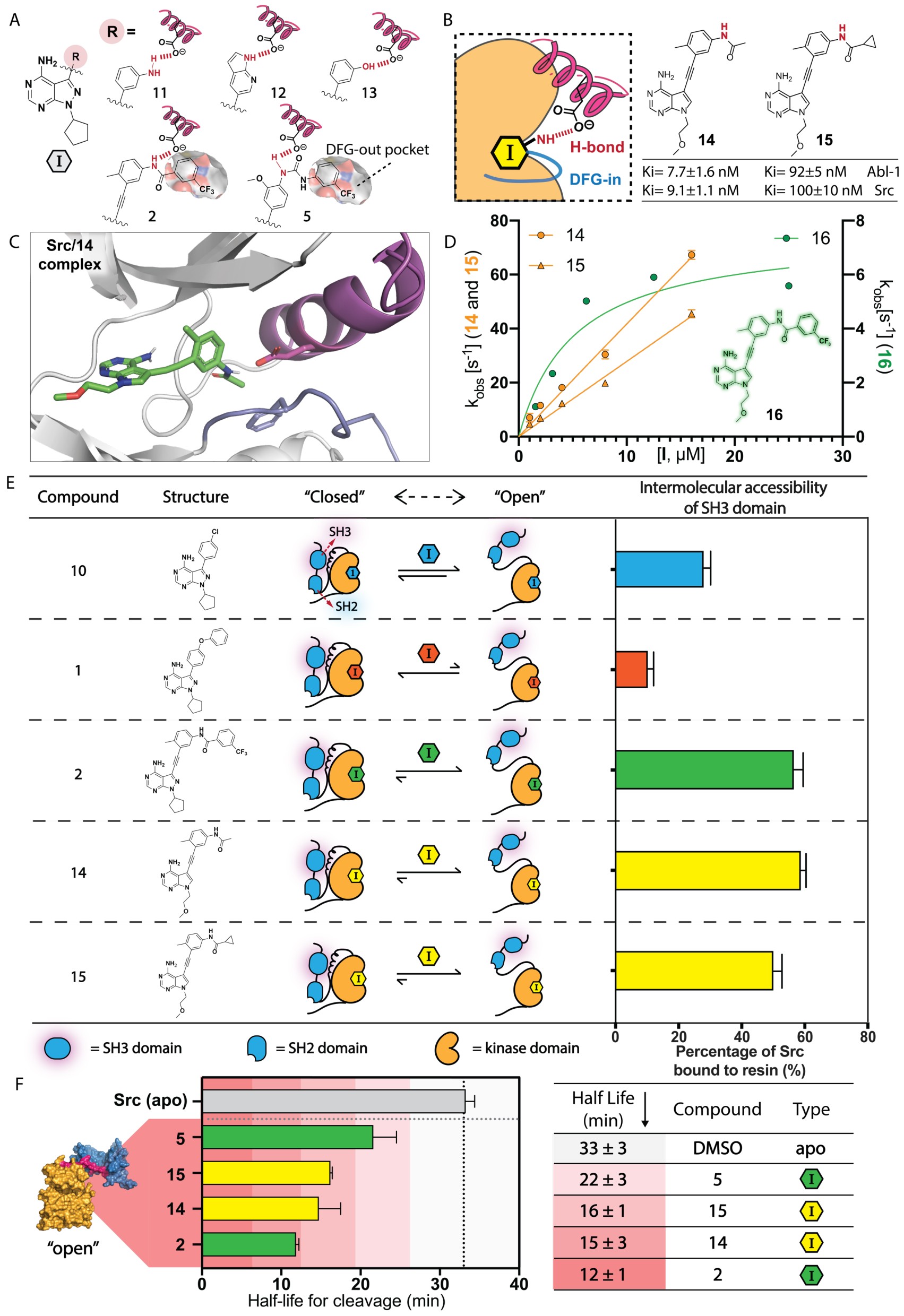


Figure 4. Analysis of how helix $\alpha \mathrm{C}$ interactions influence global conformation. (A) ATP-binding site contacts of substituents that promote an open global conformation of Src. H-bonding interactions are colored in red. The hydrophobic DFG-out pocket is annotated. (B) Structures and the predicted binding features of inhibitors $\mathbf{1 4}$ and $\mathbf{1 5}$. $\mathrm{K}_{i}$ values are shown as mean $\pm \mathrm{SEM}(\mathrm{n}=3)$. (C) X-ray cocrystal structure of the Src/14 complex (PDB ID: 6WIW). Helix $\alpha \mathrm{C}$ is colored in magenta. The activation loop is colored in blue. (D) The observed rate constants

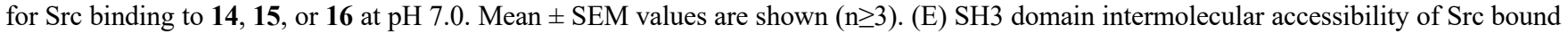
to inhibitors 10, 1, 2, 14, or 15. Mean \pm SEM are shown ( $n=3)$. (F) Thermolysin assays with Src/14 and Src/15 complexes. The half-lives for cleavage of Src's SH2-CD linker are shown as the mean \pm SEM $(n=3)$.

A

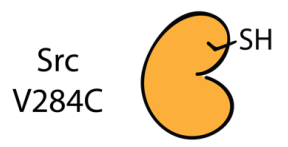

Src WT

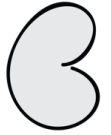

B

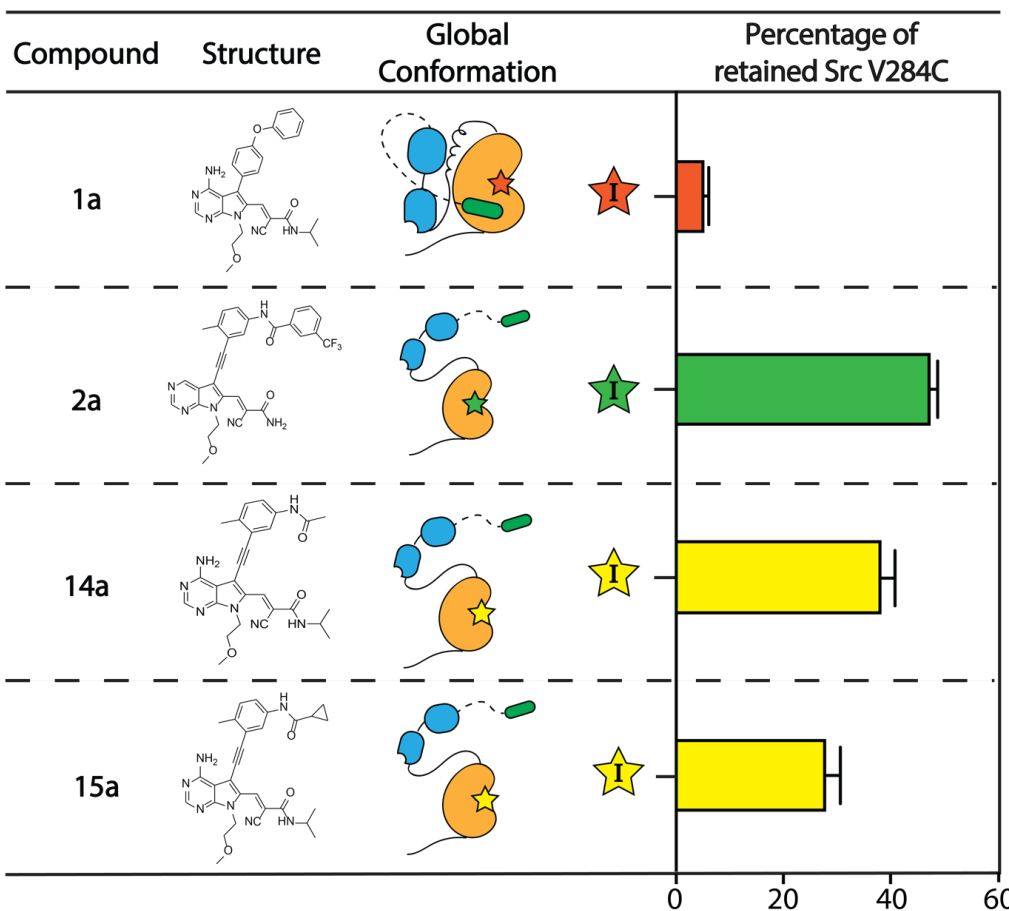

Potent

Inhibition

resistant $\rightarrow$ No

\section{V284}

SrC EVKLGQGCFGE V WMG... IV T EYM

EphA2 QKVIGAGEFGE V YKG... I I T EYM

JNK2 LKPIGSGAQGI V CAA... LV M ELM

BTK LKELGTGQFGV V KYG... II T EYM

PAK1 FEKIGQGASGT V YTA...VV M EYL
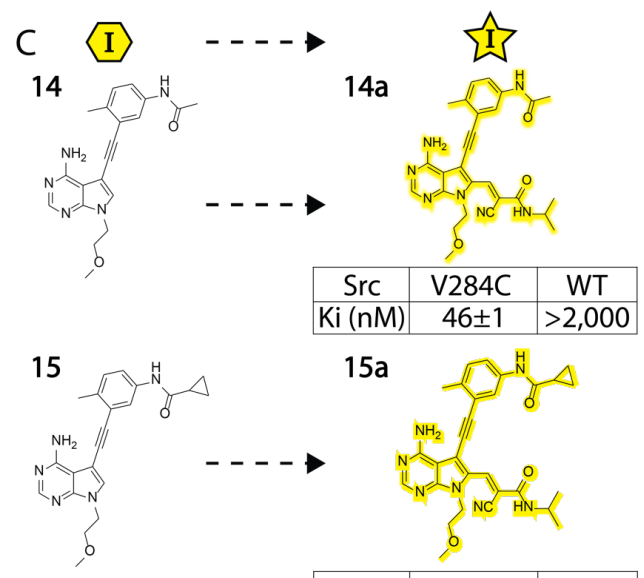

$15 a$

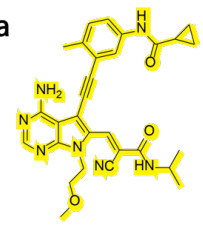

\begin{tabular}{l|l|l|} 
Src & V284C & WT \\
\hline
\end{tabular}

Ki (nM) $7.7 \pm 0.7>2,000$

$\mathrm{F}$

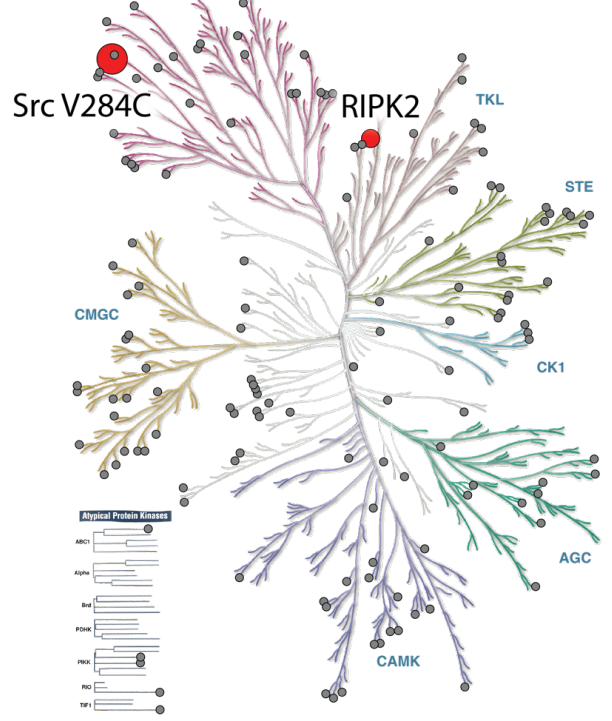

Drug resistant

Drug sensitive

-

0

$\log _{2} R<1.5 \quad \log _{2} R=2.0 \quad 3.0 \quad 4.0$

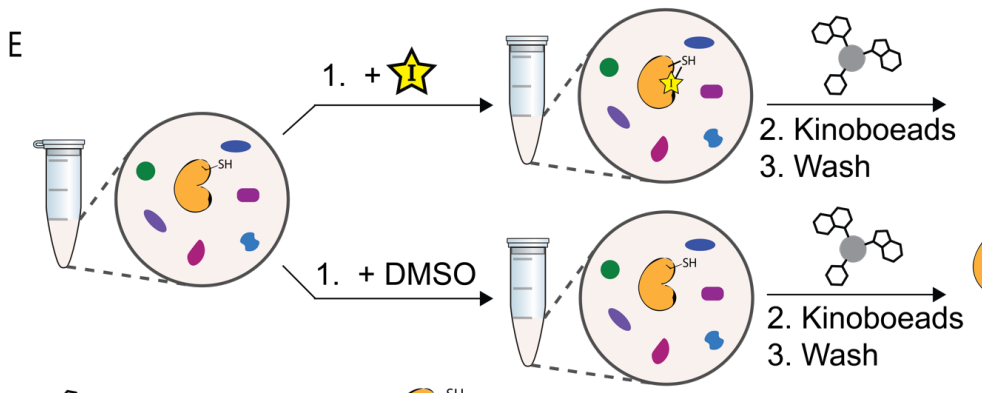

$$
\sigma_{B}=\text { Kinobeads }
$$

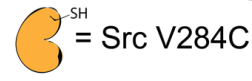

$=$ Kinases

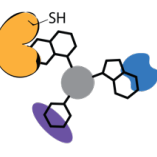

$\underset{\text { 5. Western-blot }}{\stackrel{\text { Elute }}{\longrightarrow}}$

Detection

of Src V284C

Figure 5. Chemical genetic strategy for investigating the effects of stabilizing the helix $\alpha \mathrm{C}$-in conformation of Src in cells. (A) Cysteine Installation for Modulating Allostery and Targeted Inhibition of Kinases (CystIMATIK). CystIMATIK probes provide potent inhibition of drug-sensitized kinase variants, while wild type kinases are largely resistant to CystIMATIK probes. (B) Sensitization of Src to CystIMATIK probes through the introduction of a cysteine at V284. The sequence alignment shows a conserved valine at the position equivalent to V284 
of Src. (C) Electrophile-containing analogs of 14 and 15. The Ki values of 14a (top) and 15a (bottom) for wild-type Src and Src V284C are shown as mean \pm SEM ( $n=3$ ). (D) SH3 domain pull-down assays for characterizing the global conformation of the 14a/Src V284C and 15a/ Src V284C complexes. Values shown are the mean \pm SEM $(n=3)$. (E) Kinobead-based profiling method for determining the kinome-wide selectivity of 14a and 15a. Src V284C HeLa cell lysates were incubated with DMSO, 14a, or 15a, and an immobilized matrix of nonselective kinase inhibitors (kinobeads). Captured kinases were subjected to the proteomics workflow described in the supporting information. (F) Phylogenetic trees showing the selectivity of 14a. All profiled kinases are represented by circles. Drug-sensitive kinases are shown as red circles, with the size corresponding to the level of competition (larger circle, more competed). Drug-resistant kinases are shown as gray circles. Kinases reported as being drug-sensitive $\left(\log _{2} \mathrm{R}>1.5\right)$ were also required to show significance $\left(-\log _{10} \mathrm{P}-\mathrm{Value}>1.5\right)$ from a twosample t-test with an FDR of $0.05(\mathrm{n}=3)$.

Inhibitors 14 and 15 promote an open global conformation of Src. To determine whether our new helix $\alpha \mathrm{C}$-in-stabilizing inhibitors $\mathbf{1 4}$ and $\mathbf{1 5}$ promote an open global conformation of Src's SH3-SH2-CD module, we performed SH3 domain pull-down assays and limited proteolysis experiments with inhibitor-bound Src complexes. We found that both the Src/14 and Src/15 complexes were efficiently enriched by an immobilized SH3 ligand, consistent with 14 and 15 promoting an open, regulatory domain-disengaged conformation of Src (Figure 4E). Notably, the SH3 regulatory domains of both the $\mathrm{Src} / \mathbf{1 4}$ and Src/15 complexes had similar intermolecular accessibilities as the SH3 domain of Src bound to DFG-out stabilizing inhibitor 2 . We also found that the half-lives for cleavage of the SH2-CD linkers of the Src/14 $\left(\mathrm{t}_{1 / 2}=15 \pm 3 \mathrm{~min}\right)$ and $\mathrm{Src} / \mathbf{1 5}$ $\left(\mathrm{t}_{1 / 2}=16 \pm 1 \mathrm{~min}\right)$ complexes by thermolysin was within the range of Src bound to DFG-out-stabilizing inhibitors $\mathbf{2}$ and $\mathbf{5}$ (Figure 4F). Thus, the observed capability of DFG-out-stabilizing inhibitors to promote an open global conformation of Src appears to rely on their ability to stabilize an active conformation of helix $\alpha \mathrm{C}$, rather than their ability to perturb the activation loop.

A chemical genetic strategy for investigating the effects of stabilizing the helix $\alpha \mathbf{C}$-in conformation in cells. We have recently demonstrated that modulating Src's global conformation in cells with conformation-selective inhibitors can influence the phosphotransferase-independent functions of its regulatory domains. ${ }^{13-14}$ To determine how stabilizing the helix $\alpha \mathrm{C}$ in conformation (characterized by an inward position of the helix $\alpha \mathrm{C}$ in the N-lobe and an unperturbed activation loop) of Src affects its phosphotransferase-independent functions in cells compared to alternative ATP-binding site conformations, we pursued a chemical genetic strategy for sensitizing kinases to specific inhibtors. ${ }^{35-36,39-40}$ Our strategy, which we call Cystine Installation for Modulating Allostery and Target Inhibition of Kinases (CystIMATIK) (Figure 5A) ${ }^{14}$ involves the introduction of a cysteine residue at the $\beta 2$ strand of the $\mathrm{N}$-terminal lobe of the catalytic domains of kinases that provides sensitivity to pyrrolopyrimidine-based inhibitors that contain a Michael acceptor at the C-6 position (Figure 5B). To implement the CystIMATIK strategy for Src, we generated electrophile-containing analogs of 14 and 15 (14a and 15a, Figure 5C) and tested them for inhibition of drug-sensitized Src (Src V284C). We found that both 14a and 15a provided potent inhibition of Src V284C but not the wild-type kinase (Figure 5C).

To validate that both 14a and 15a modulate the global conformation of Src like their non-electrophilic counterparts (Figure 5D), we performed SH3 domain pull-down assays with inhibitor-bound Src V284C complexes. As expected, we found that the SH3 domain of the Src V284C/1a complex was largely inaccessible to an immobilized $\mathrm{SH} 3$ ligand while the Src V284C/2a complex's SH3 domain readily participated in intermolecular interactions. Like the Src V284C/2a complex, we observed that both the Src V284C/14a and Src V284C/15a complexes were efficiently enriched by the immobilized SH3 ligand, which is consistent with their ability to promote an open global conformation of Src V284C's SH3-SH2-CD module.

Prior to studying how 14a and 15a affect Src's phosphotransferase-independent functions in cells, we determined their selectivity for Src V284C with a lysate profiling method (Figure 5E). ${ }^{41-43}$ To do this, we measured the ability of $\mathbf{1 4 a}$ and $\mathbf{1 5 a}$ to compete for binding to a mixture of resin-immobilized nonselective ATP-competitive inhibitors (kinobeads) with lysate kinases. The binding of an inhibitor being profiled prevents enrichment of lysate kinases by kinobeads, which allows us to profile inhibitors against a large number of human kinases using quantitative mass spectrometry. Specifically, inhibitor selectivity can be determined by measuring the loss of relative enrichment of kinases in inhibitor-treated lysates versus lysate treated with a vehicle control (DMSO). 14a and 15a were both profiled at a single high concentration $(20 \mu \mathrm{M})$ in Src V284C-expressing HeLa lysates and the relative enrichment of $\sim 150$ kinases was quantified. We found that Src V284C was the most competed target of 14a, with Receptor Interacting Serine/Threonine Kinase 2 (RIPK2) being the only significant off-target kinase (Figure 5F). 15a demonstrated a similar selectivity profile, except Src V284C and RIPK2 were competed at similar levels (Figure S10). Thus, our observation suggests that Src V284C is the primary target for 14a and 15a, with RIPK2 being the only offtarget for both inhibitors in the Src V284C-expressing HeLa lysates.

Stabilization of Src in the helix $\alpha \mathrm{C}$-in conformation is sufficient to promote a phosphotransferase-independent alteration in cell morphology. We recently showed that Src can promote non-apoptotic membrane blebs, which are characterized by localized disruption of the actin-myosin cortex, through a phosphotransferase-independent mechanism and that conformation-selective inhibitors can differentially modulate this phenotype (Figure 6A) ${ }^{14,44} \mathrm{We}$ found that the expression of drugsensitive Src V284C and wild-type Src (WT) in HeLa cells yielded a basal number of cells with membrane blebs $(\sim 10 \%)$, which was negligibly influenced by treatment with helix $\alpha \mathrm{C}$ out-stabilizing 1a (Figure 6B). In contrast, treatment with DFG-out-stabilizing inhibitor 2a significantly induced membrane blebs in HeLas expressing Src V284C ( 55\%) but not in Src WT-expressing HeLa cells (Figure 6B). ${ }^{14}$

Our observations with $\mathbf{1 4}$ and $\mathbf{1 5}$ in vitro made us interested in investigating whether stabilizing Src in the helix $\alpha C$-in conformation in cells is sufficient to mimic the phenotype promoted by DFG-out-stabilizing inhibitor 2a. Therefore, we tested the influence of 14a and 15a on the cell morphology of Src V284C-expressing HeLas. We found that treatment of Src V284C-expressing HeLas with 14a and 15a yielded a significant increase in the percentage of cells with membrane blebs 
A
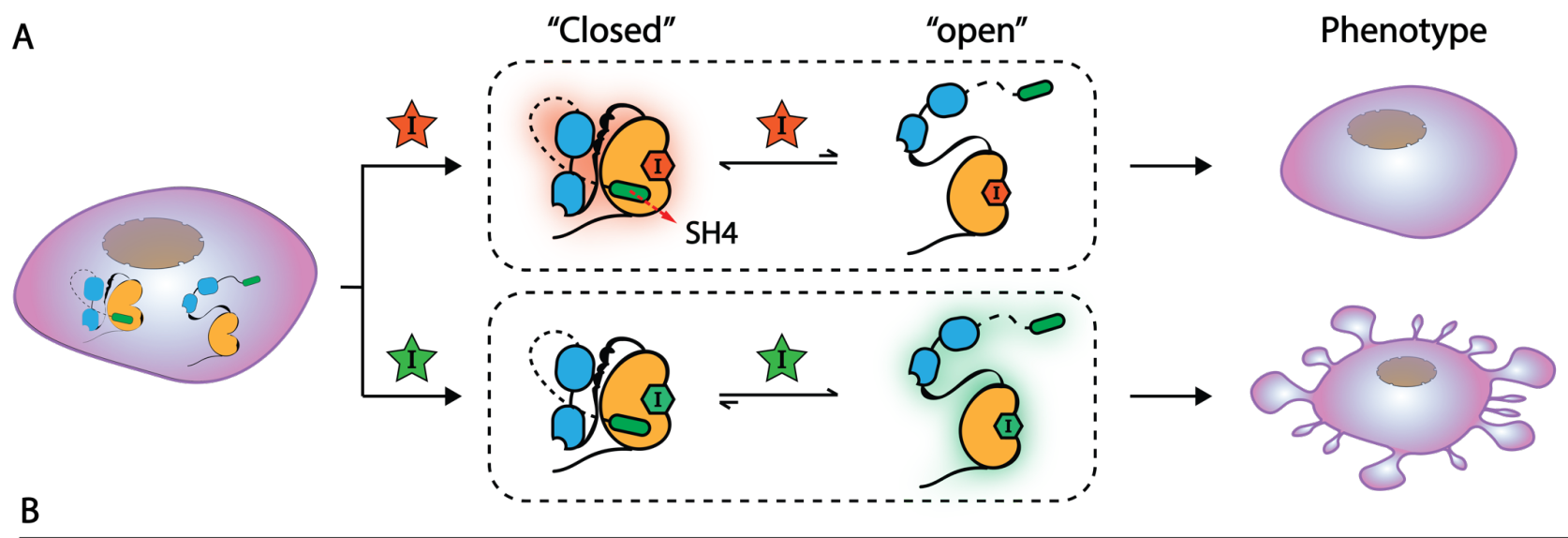

"Closed"

"open"

Phenotype

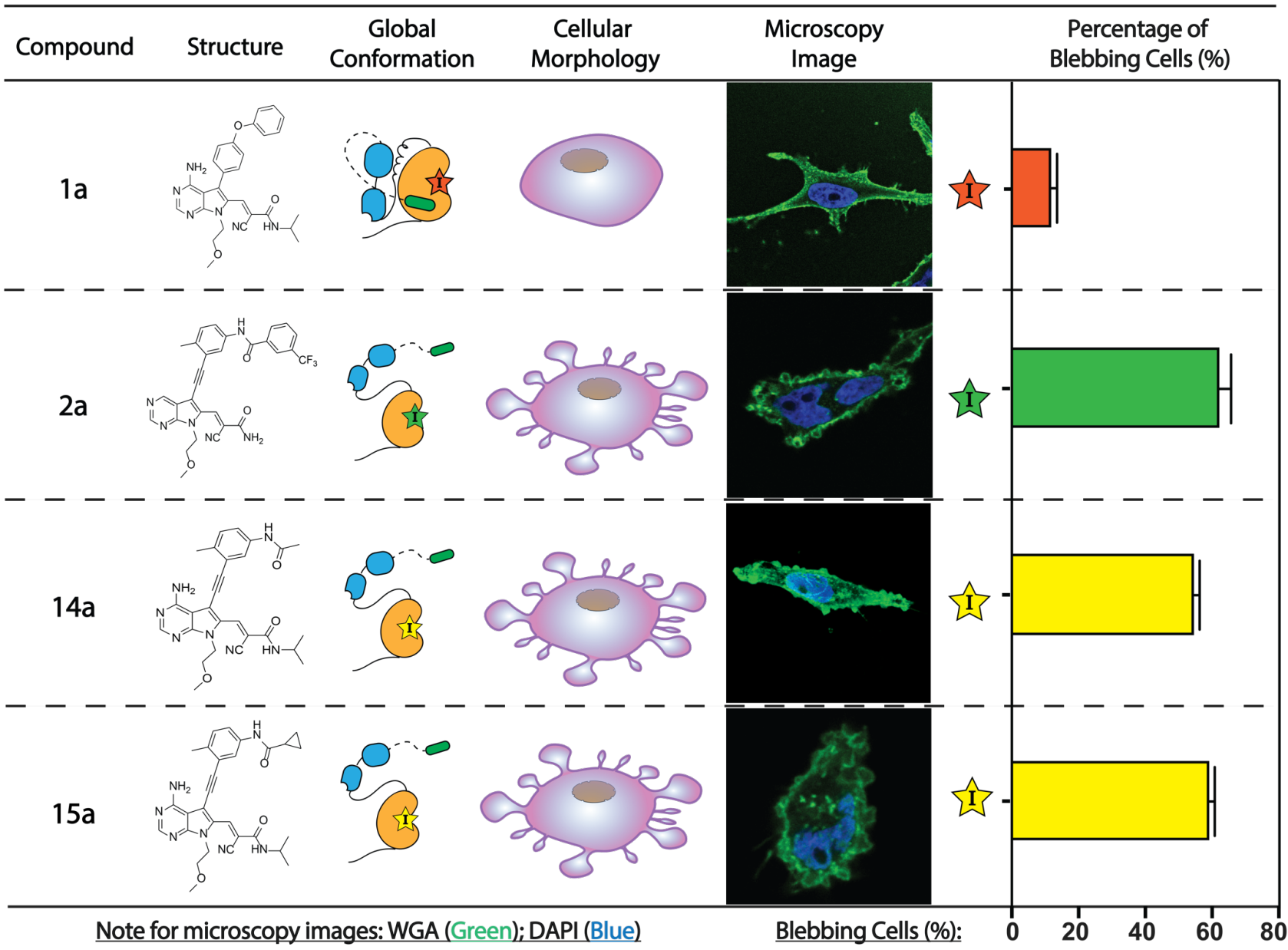

Figure 6. Stabilization of Src's helix $\alpha \mathrm{C}$ in an active conformation (helix $\alpha \mathrm{C}$-in) is sufficient to promote a Src-mediated, phosphotransferaseindependent alteration in cell morphology. (A) CystIMATIK probes that enforce an open global conformation of Src V284C promote nonapoptotic membrane blebs in HeLas expressing drug-sensitized Src V284C. (B) Levels of membrane blebs in Src V284C-expressing HeLas that are treated with CystIMATIK probes. Percentages of blebbing cells are shown as mean \pm SEM ( $n=3$ ). Nuclei were stained with DAPI (blue) and membranes were stained with Wheat Germ Agglutinin (WGA)-Alexa488 (green). For each replicate, 17 to 32 images were taken and the percentage of blebbing cells from all imaged cells was quantified. Each image contained 1-3 stained cells. The number of blebbing cells was scored based on the criteria described in the supporting information. 

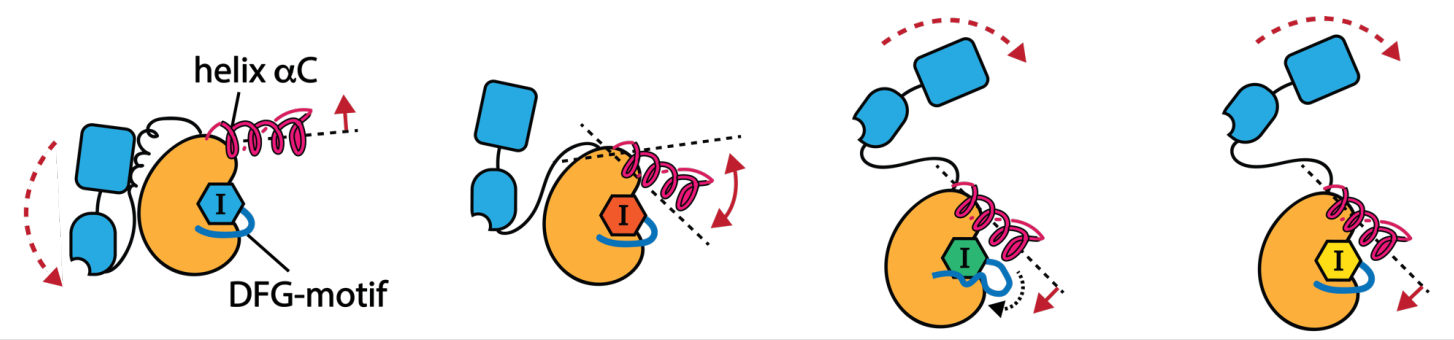

\begin{tabular}{cc}
\hline $\begin{array}{c}\text { ATP-Binding Site } \\
\text { Conformation }\end{array}$ & Helix $\alpha \mathrm{C}$-out \\
\hline DFG-motif & IN \\
Helix $\alpha C$ & OUT \\
Global & Closed \\
Conformation & \multicolumn{2}{c}{$\square=\mathrm{SH}$ domain }
\end{tabular}

Neutral

DFG-out

Helix $\alpha C$-in

Figure 7. ATP binding site contacts of inhibitors that allosterically modulate the SH3-SH2-CD module. An inhibitor's ability to stabilize helix $\alpha \mathrm{C}$ in an active conformation (helix $\alpha \mathrm{C}$-in) is the main determinant of its ability to promote an open global conformation of the SH3$\mathrm{SH} 2-\mathrm{CD}$ module.

( $60 \%$ ) (Figure 6B), while Src WT-expressing HeLas remained at the basal level when treated with these inhibitors (Figure S11). Notably, Src V284C-expressing HeLas that are treated with 14a and 15a showed levels of blebbing comparable to cells treated with $\mathbf{2 a}$. Thus, stabilization of Src in the helix $\alpha \mathrm{C}$-in conformation is sufficient to induce membrane blebs. Therefore, the observed ability of a DFG-out-stabilizing inhibitor to promote membrane blebs is not due to its ability to perturb Src's activation loop but rather its ability to stabilize Src's helix $\alpha \mathrm{C}$ in an active conformation.

\section{CONCLUSIONS}

Interdomain regulation is a defining characteristic of multidomain kinases. Almost half of the human kinases contain at least one auxiliary domain, which, in many cases, are implicated in phosphotransferase-independent functions within cells. ${ }^{5}$ Although a growing body of evidence strongly suggests that conformation-selective, ATP-competitive inhibitors can modulate regions distal to a kinase's $\mathrm{CD}$, the overall generality and molecular determinants of this phenomenon are not completely known. In this study, we have demonstrated that inhibitors which stabilize two different inactive ATP-binding site conformations can divergently modulate the global conformation of multidomain tyrosine kinases that contain a Src-like regulatory SH3-SH2-CD module, including the SFKs, Abl-1, and the Tec family kinase BTK. Despite structural differences in the N- and C-termini of SFKs, Abl-1, and BTK, the allosteric communication between their ATP-binding sites and regulatory $\mathrm{SH} 3$ and SH2 domains remain conserved. This observation suggests that the effects of conformation-selective inhibitors on global conformation are likely conserved in other tyrosine kinases that contain a homologous $\mathrm{SH}-\mathrm{SH} 2-\mathrm{CD}$ module.

By systematically analyzing the common features of inhibitors that promote an open global conformation of Src, we were able to develop chemical probes that allowed us to isolate the effects of specific ATP-binding site interactions. With these probes, we found that an inhibitor's ability to promote an open global conformation of Src's SH3-SH2-CD module mainly relies on its ability to stabilize an active conformation of the helix $\alpha \mathrm{C}$. Thus, the noted ability of DFG-out-stabilizing inhibitors to promote an open global conformation in Src and Abl-1 is most likely due to their interactions with the helix $\alpha \mathrm{C}$, rather than perturbation of the DFG-motif in the activation loop (Figure 7). Furthermore, we have determined that stabilization of Src's helix $\alpha \mathrm{C}$ in the active conformation (helix $\alpha \mathrm{C}$-in) is sufficient to promote a Src-mediated, phosphotransferase-independent alteration in cell morphology, a phenotypic effect consistent with releasing the otherwise intramolecularly sequestered N-terminal SH4 domain of Src. Like Src's SH4 domain, Abl-1's N-terminal myristate and BTK's N-terminal PH-TH domain not only suppress phosphotransferase activity by interacting with their $\mathrm{CDs}^{26-27,45-46}$ but also interact with the plasma membrane and additional organelles when intermolecularly accessible. ${ }^{27,45,47-}$

${ }^{49}$ Based on our observation with Src, inhibitors that stabilize or perturb the helix $\alpha \mathrm{Cs}$ of Abl-1 and BTK likely influence the localization and other phosphotransferase-independent functions of these kinases by allosterically modulating global conformation. ${ }^{50-51}$ Taken together, our results have direct implications for targeting the helix $\alpha \mathrm{C}$ in the ATP-binding site of multidomain tyrosine kinases with inhibitors to modulate kinase functional surfaces distal to their CDs. Although our results highlight the effects of conformation-selective inhibitors on the subset of tyrosine kinases that contain an SH3-SH2-CD module, it is likely that multidomain kinases with alternative regulatory architectures can also be allosterically modulated using similar principles. The conceptual framework and probes that we describe in this report may help facilitate these studies.

\section{ASSOCIATED CONTENT}

\section{Supporting Information}

The Supporting Information is available free of charge on the ACS Publications website.

- Supplementary figures, experimental details and synthesis of all compounds used in the study (PDF)

- Supplementary Excel File, containing the imputed MaxQuant output data for inhibitor profiling of 14a and 15a (XLSX)

\section{AUTHOR INFORMATION}

\section{Corresponding Author}

* E-mail: djmaly@uw.edu 


\section{ORCID}

Linglan Fang: 0000-0003-2637-090X

Dustin J. Maly: 0000-0003-0094-0177

\section{Notes}

The authors declare no competing financial interest.

\section{ACKNOWLEDGMENT}

We thank Nathaniel Peters at the W.M. Keck Center for Advanced Studies in Neural Signaling for microscopy assistance. We thank Priska D. von Haller at the University of Washington Proteomics Resource (UWPR) and Martin Sadilek for mass spectrometry assistance. We thank Stephen Taylor for providing the Flp-In T-Rex HeLa cell line. We thank NSLS2 facility of U.S. Department of Energy (DOE) Office operated by the Brookhaven National Laboratory under Contract No. DE-SC0012704. This work was supported by NIH R01 GM086858 (DJM), NIH R35 GM119437 (MAS), and R25 GM103962 (JVB).

\section{ABBREVIATIONS}

SFKs, Src-family kinases; CD, catalytic domain; 3D, three domain kinase containing $\mathrm{CD}, \mathrm{SH} 2$ and $\mathrm{SH} 3$ domain; CystIMATIK, Cystine Installation for Modulating Allostery and Targeted Inhibition of Kinases; DMSO, dimethyl sulfoxide; NMR, nuclear magnetic resonance; MS, mass spectrometry

\section{REFERENCES}

(1) Taylor, S. S.; Keshwani, M. M.; Steichen, J. M.; Kornev, A. P., Philos Trans R Soc Lond B Biol Sci 2012, 367 (1602), 2517-28.

(2) Manning, G.; Whyte, D. B.; Martinez, R.; Hunter, T.; Sudarsanam, S., Science 2002, 298 (5600), 1912-34.

(3) Kuriyan, J.; Eisenberg, D., Nature 2007, 450 (7172), 983-90.

(4) Pellicena, P.; Kuriyan, J., Curr Opin Struct Biol 2006, 16 (6), 702-9.

(5) Kung, J. E.; Jura, N., Structure 2016, 24 (1), 7-24.

(6) Huse, M.; Kuriyan, J., Cell 2002, 109 (3), 275-82.

(7) Taylor, S. S.; Kornev, A. P., Trends Biochem Sci 2011, 36 (2), 65-77.

(8) Engen, J. R.; Wales, T. E.; Hochrein, J. M.; Meyn, M. A., 3rd; Banu Ozkan, S.; Bahar, I.; Smithgall, T. E., Cell Mol Life Sci 2008, 65 (19), 3058-73.

(9) Sicheri, F.; Kuriyan, J., Curr Opin Struct Biol 1997, 7 (6), 777 85.

(10) Kim, L. C.; Song, L.; Haura, E. B., Nat Rev Clin Oncol 2009, $6(10), 587-95$.

(11) Jura, N.; Zhang, X.; Endres, N. F.; Seeliger, M. A.; Schindler, T.; Kuriyan, J., Mol Cell 2011, 42 (1), 9-22.

(12) Boggon, T. J.; Eck, M. J., Oncogene 2004, 23 (48), 7918-27.

(13) Fang, L.; Chakraborty, S.; Dieter, E. M.; Potter, Z. E.; Lombard, C. K.; Maly, D. J., J Am Chem Soc 2019, 141 (30), 11912-11922.

(14) Ahler, E.; Register, A. C.; Chakraborty, S.; Fang, L.; Dieter, E. M.; Sitko, K. A.; Vidadala, R. S. R.; Trevillian, B. M.; Golkowski, M.; Gelman, H.; Stephany, J. J.; Rubin, A. F.; Merritt, E. A.; Fowler, D. M.; Maly, D. J., Mol Cell 2019, 74 (2), 393-408 e20.

(15) Young, M. A.; Gonfloni, S.; Superti-Furga, G.; Roux, B.; Kuriyan, J., Cell 2001, 105 (1), 115-26.

(16) Xu, W.; Doshi, A.; Lei, M.; Eck, M. J.; Harrison, S. C., Mol Cell 1999, 3 (5), 629-38.

(17) Chakraborty, S.; Inukai, T.; Fang, L.; Golkowski, M.; Maly, D. J., ACS Chem Biol 2019, 14 (6), 1249-1259.

(18) Leonard, S. E.; Register, A. C.; Krishnamurty, R.; Brighty, G. J.; Maly, D. J., ACS Chem Biol 2014, 9 (8), 1894-905.
(19) Krishnamurty, R.; Brigham, J. L.; Leonard, S. E.; Ranjitkar, P.; Larson, E. T.; Dale, E. J.; Merritt, E. A.; Maly, D. J., Nat Chem Biol 2013, 9 (1), 43-50.

(20) Register, A. C.; Leonard, S. E.; Maly, D. J., Biochemistry 2014, 53 (44), 6910-23.

(21) Tong, M.; Pelton, J. G.; Gill, M. L.; Zhang, W.; Picart, F.; Seeliger, M. A., Nat Commun 2017, 8 (1), 2160.

(22) Agius, M. P.; Ko, K. S.; Johnson, T. K.; Kwarcinski, F. E.; Phadke, S.; Lachacz, E. J.; Soellner, M. B., ACS Chem Biol 2019, 14 (7), 1556-1563.

(23) Kwarcinski, F. E.; Brandvold, K. R.; Phadke, S.; Beleh, O. M.; Johnson, T. K.; Meagher, J. L.; Seeliger, M. A.; Stuckey, J. A.; Soellner, M. B., ACS Chem Biol 2016, 11 (5), 1296-304.

(24) Seeliger, M. A.; Nagar, B.; Frank, F.; Cao, X.; Henderson, M. N.; Kuriyan, J., Structure 2007, 15 (3), 299-311.

(25) Skora, L.; Mestan, J.; Fabbro, D.; Jahnke, W.; Grzesiek, S., Proc Natl Acad Sci U S A 2013, 110 (47), E4437-45.

(26) Hantschel, O.; Nagar, B.; Guettler, S.; Kretzschmar, J.; Dorey, K.; Kuriyan, J.; Superti-Furga, G., Cell 2003, 112 (6), 845-57.

(27) Nagar, B.; Hantschel, O.; Young, M. A.; Scheffzek, K.; Veach, D.; Bornmann, W.; Clarkson, B.; Superti-Furga, G.; Kuriyan, J., Cell 2003, 112 (6), 859-71.

(28) Nagar, B.; Hantschel, O.; Seeliger, M.; Davies, J. M.; Weis, W. I.; Superti-Furga, G.; Kuriyan, J., Mol Cell 2006, 21 (6), 78798.

(29) Sonti, R.; Hertel-Hering, I.; Lamontanara, A. J.; Hantschel, O.; Grzesiek, S., J Am Chem Soc 2018, 140 (5), 1863-1869.

(30) Amatya, N.; Lin, D. Y.; Andreotti, A. H., Biochem Soc Trans 2019, 47 (4), 1101-1116.

(31) Tong, M.; Seeliger, M. A., ACS Chem Biol 2015, 10 (1), 190200.

(32) Dar, A. C.; Lopez, M. S.; Shokat, K. M., Chem Biol 2008, 15 (10), 1015-22.

(33) MacAuley, A.; Cooper, J. A., Mol Cell Biol 1989, 9 (6), 264856.

(34) Apsel, B.; Blair, J. A.; Gonzalez, B.; Nazif, T. M.; Feldman, M. E.; Aizenstein, B.; Hoffman, R.; Williams, R. L.; Shokat, K. M.; Knight, Z. A., Nat Chem Biol 2008, 4 (11), 691-9.

(35) Garske, A. L.; Peters, U.; Cortesi, A. T.; Perez, J. L.; Shokat, K. M., Proc Natl Acad Sci U S A 2011, 108 (37), 15046-52.

(36) Zhang, C.; Lopez, M. S.; Dar, A. C.; Ladow, E.; Finkbeiner, S.; Yun, C. H.; Eck, M. J.; Shokat, K. M., ACS Chem Biol 2013, 8 (9), 1931-8.

(37) Seeliger, M. A.; Ranjitkar, P.; Kasap, C.; Shan, Y.; Shaw, D. E.; Shah, N. P.; Kuriyan, J.; Maly, D. J., Cancer Res 2009, 69 (6), 2384-92.

(38) Shan, Y.; Seeliger, M. A.; Eastwood, M. P.; Frank, F.; Xu, H.; Jensen, M. O.; Dror, R. O.; Kuriyan, J.; Shaw, D. E., Proc Natl Acad Sci U S A 2009, 106 (1), 139-44.

(39) Kung, A.; Schimpl, M.; Ekanayake, A.; Chen, Y. C.; Overman, R.; Zhang, C., ACS Chem Biol 2017, 12 (6), 1499-1503. (40) Zhang, C.; Kenski, D. M.; Paulson, J. L.; Bonshtien, A.; Sessa, G.; Cross, J. V.; Templeton, D. J.; Shokat, K. M., Nat Methods 2005, 2 (6), 435-41.

(41) Bantscheff, M.; Eberhard, D.; Abraham, Y.; Bastuck, S.; Boesche, M.; Hobson, S.; Mathieson, T.; Perrin, J.; Raida, M.; Rau, C.; Reader, V.; Sweetman, G.; Bauer, A.; Bouwmeester, T.; Hopf, C.; Kruse, U.; Neubauer, G.; Ramsden, N.; Rick, J.; Kuster, B.; Drewes, G., Nat Biotechnol 2007, 25 (9), 1035-44.

(42) Golkowski, M.; Brigham, J. L.; Perera, G. K.; Romano, G. E.; Maly, D. J.; Ong, S. E., Medchemcomm 2014, 5 (3), 363-369.

(43) Golkowski, M.; Perera, G. K.; Vidadala, V. N.; Ojo, K. K.; Van Voorhis, W. C.; Maly, D. J.; Ong, S. E., Mol Omics 2018, 14 (1), 26-36

(44) Charras, G. T., J Microsc 2008, 231 (3), 466-78.

(45) Wang, Q.; Pechersky, Y.; Sagawa, S.; Pan, A. C.; Shaw, D. E., Proc Natl Acad Sci U S A 2019, 116 (19), 9390-9399. 
(46) Wang, Q.; Vogan, E. M.; Nocka, L. M.; Rosen, C. E.; Zorn, J. A.; Harrison, S. C.; Kuriyan, J., Elife 2015, 4.

(47) Choi, Y.; Seeliger, M. A.; Panjarian, S. B.; Kim, H.; Deng, X.; Sim, T.; Couch, B.; Koleske, A. J.; Smithgall, T. E.; Gray, N. S., J Biol Chem 2009, 284 (42), 29005-14.

(48) Hantschel, O., Genes Cancer 2012, 3 (5-6), 436-46.

(49) Varnai, P.; Rother, K. I.; Balla, T., J Biol Chem 1999, 274 (16), 10983-9.
(50) Morita, S.; Villalta, S. A.; Feldman, H. C.; Register, A. C.; Rosenthal, W.; Hoffmann-Petersen, I. T.; Mehdizadeh, M.; Ghosh, R.; Wang, L.; Colon-Negron, K.; Meza-Acevedo, R.; Backes, B. J.; Maly, D. J.; Bluestone, J. A.; Papa, F. R., Cell Metab 2017, 25 (4), 883-897 e8.

(51) Saito, K.; Tolias, K. F.; Saci, A.; Koon, H. B.; Humphries, L. A.; Scharenberg, A.; Rawlings, D. J.; Kinet, J. P.; Carpenter, C. L., Immunity 2003, 19 (5), 669-78. 